\title{
Accelerate the ePC-SAFT-DFT calculation with the Chebyshev pseudo-spectral collocation method
}

\author{
Yunhao Sun ${ }^{\mathrm{a}, \mathrm{b}}$, Xiaohua Lu ${ }^{\mathrm{b}}$, Gulou Shen ${ }^{\mathrm{c},}$, Xiaoyan Jia,*
}

aDivision of Energy Science/Energy Engineering, Luleå University of Technology, 97187 Luleå, Sweden

bState Key Laboratory of Materials-Oriented Chemical Engineering, Nanjing Tech University, Nanjing 210009, P. R. China

'National \& Local Joint Engineering Research Center for Deep Utilization Technology of Rocksalt Resource, Faculty of Chemical Engineering, Huaiyin Institute of Technology, Huaian 223003, China

\section{AUTHOR INFORMATION}

\section{Corresponding Authors}

*E-mail: xiaoyan.ji@ltu.se.

*E-mail: 1sheng@hyit.edu.cn. 


\section{External potential used in this work}

For a slit pore with a width $H$, the planar 9-3 potential $^{1}$ or Lee's $10-4$ Lennard-Jones potential ${ }^{2,3}$ can be used:

$U_{s, 9-3, i}(z)=\frac{2 \pi \rho_{a t o m} \sigma_{s i}^{3} \varepsilon_{s i}}{3}\left[\frac{2}{15}\left(\frac{\sigma_{s i}}{z}\right)^{9}-\left(\frac{\sigma_{s i}}{z}\right)^{3}\right]$

$U_{s, 10-4, i}(z)=2 \pi \rho_{a t o m} \varepsilon_{s i} \sigma_{s i}^{2}\left[\frac{2}{5}\left(\frac{\sigma_{s i}}{z}\right)^{10}-\sum_{i=1}^{4} \frac{\sigma_{s i}^{4}}{(z+(i-1) \Delta)^{4}}\right]$

where $z$ is the distance between the surface and fluid molecule, $\rho_{\text {atom }}$ is the solid atom density, $\sigma_{s i}$ is the effective solid-fluid diameter, $\Delta$ is the distance between the atomic layer of solid, and $\varepsilon_{s i}$ is the potential representing the interaction between surface and fluid segment.

The total interaction potential between fluid and both walls of the slit is obtained by:

$V(z)=U(z)+U(H-z)$

For a cylindrical pore with radius $R$, the cylindrical 9-3 potential can be used: ${ }^{4}$

$V(r, R)=2 \pi \rho_{a t o m} \varepsilon_{s i} \sigma_{s i}^{3}\left[\Phi_{6}\left(r, R, \sigma_{s i}\right)-\Phi_{3}\left(r, R, \sigma_{s i}\right)\right]$

with

$\Phi_{6}\left(r, R, \sigma_{s i}\right)=\frac{4 \sqrt{\pi} \Gamma\left(n-\frac{1}{2}\right)}{2 n-3 \Gamma(n)}\left(\frac{\sigma_{s i}}{R}\right)^{2 n-3}\left[1-\left(\frac{r}{R}\right)^{2}\right]^{3-2 n} \boldsymbol{F}\left[\frac{3-2 n}{2}, \frac{5-2 n}{2} ; 1 ;\left(\frac{r}{R}\right)^{2}\right]$

where $r$ is the fluid molecule's distance from the center of the cylindrical pore, $\Gamma$ is the Gamma function, and $\boldsymbol{F}$ is the hypergeometric function.

The size $\left(\sigma_{s i}\right)$ and energy $\left(\varepsilon_{s i}\right)$ parameters of solid-fluid interaction can be set as adjustable parameters in modeling or evaluating with Berthelot-Lorentz combining rules:

$\left\{\begin{array}{l}\sigma_{s i}=\frac{\sigma_{i}+\sigma_{s}}{2} \\ \varepsilon_{s i}=\sqrt{\varepsilon_{i} \varepsilon_{s}}\end{array}\right.$

where $\sigma_{s}$ and $\varepsilon_{s}$ are the size and potential parameters of a solid surface. 
$\left\{\begin{array}{c}I_{1}{ }^{c}=2 \pi R_{c} \int_{-R_{c}}^{R_{c}} d z^{\prime} f\left(z+z^{\prime}\right) \\ I_{2}{ }^{c}=2 \pi \overrightarrow{\boldsymbol{e}}_{z} \int_{-R_{c}}^{R_{c}} d z^{\prime} f\left(z+z^{\prime}\right) z^{\prime} \\ I_{3}{ }^{c}=\pi \int_{-R_{c}}^{R_{c}} d z^{\prime} f\left(z+z^{\prime}\right)\left(R_{c}{ }^{2}-z^{\prime 2}\right)\end{array}\right.$

where $\overrightarrow{\boldsymbol{e}}_{z}$ denotes the unity vector in the $z$-direction.

\section{Proof of equation (11)}

For $I_{3}{ }^{c}$, the integral region is a sphere with a diameter $R_{c}$. In order to apply the Chebyshev pseudo-spectral collocation method for the special case, the integral region outside the cylindrical pore should be excluded. A typical 3D view of the region to be integrated in $I_{3}{ }^{c}$ for the special case is illustrated in Figure S1.

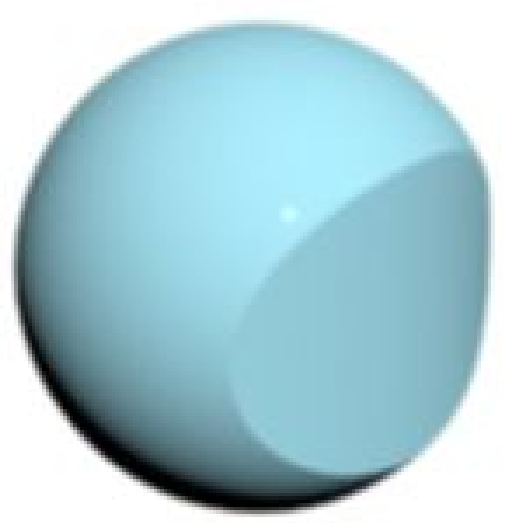

Figure S1. Typical 3D view of the space to be integrated in $I_{3}{ }^{c}$ for the special case 


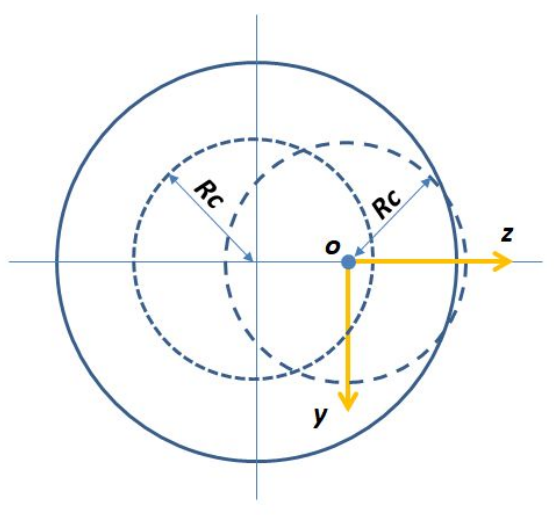

Figure S2. Schematic diagram of the local coordinate

In order to evaluate the integration over this region, following the work by Gerstenmaier et al., 5 a local coordinate was developed with the origin placed at the center of the sphere $o$. The $z$-axis is perpendicular to the cylinder's pointing outward in the radial direction and the $y$ axis is parallel to the cylinder's axis, and the $x$-axis is correspondingly oriented (Figure S2). Adopting spherical coordinates with respect to this local reference system, (i.e., the azimuthal angle $\varphi$ is located in $x y$ plane) the integration can be represented with a triple integral. The integral region can split into two sub-regions, as illustrated in Figure S3. Therefore, the $I_{3}{ }^{c}$ can be represented with two integrals, which correspond to two sub-regions and consider symmetrical, and equation (11a) can be obtained.

Region corresponding to the first integral in equation (11a)

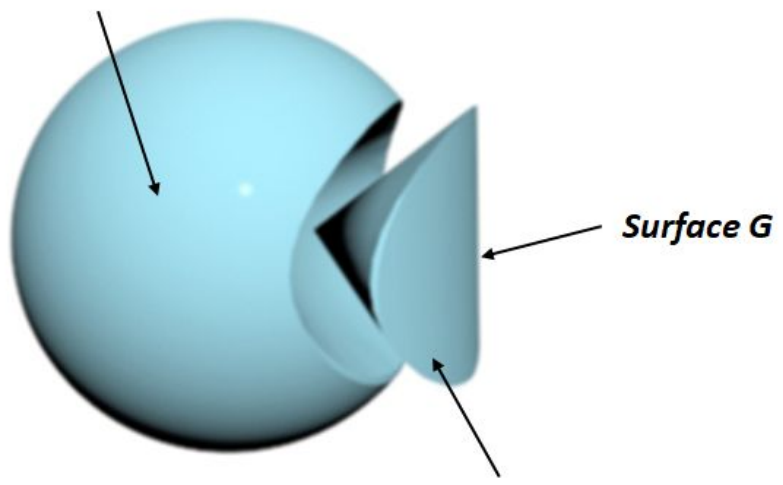

Region corresponding to the second integral in equation (11a)

Figure S3. Regions for the two integrals in equation (11a)

In equation (11a), $t=\sin \theta$ where $\theta$ represents the polar angle and $r$ ' is the distance to the cylinder's axis.

For the first integral, the upper limit of $t$ can be obtained with: 
$r^{\prime}=\sqrt{r^{2}+2 R_{c} r t+R_{c}{ }^{2}-R_{c}{ }^{2}\left(1-t^{2}\right) \cos ^{2} \varphi}=R$

The positive root of (S5) is the upper limit of $t$ in the first integral which is $f_{1}$ in equation (11b).

For the second integral, the lower limit of $t$ is exactly the same as $f_{1}$, while the upper limit of $t$ ' is the distance between the origin to the point at the surface $G$ (Figure 2) with specific $\varphi$ and $\theta$. It can be calculated with:

$r^{\prime}=\sqrt{r^{2}+2 t^{\prime} r t+t^{\prime 2}-t^{\prime 2}\left(1-t^{2}\right) \cos ^{2} \varphi}=R$

The positive root of (S6) is the upper limit of $t^{\prime}$ in the second integral, which is $f_{2}$ in equation (11b).

\section{Proof of the equations (13)}

For a cylindrical pore with a radius of $R$, the mean electric potential follows:

$\psi(r)=\psi^{w}-\int_{R}^{r} d r^{\prime} E\left(r^{\prime}\right)$

where $E$ represents Electric Field Intensity and it can be calculated with Gauss' law:

$$
\oiint \vec{E} \cdot d \vec{S}=\frac{Q}{\varepsilon_{0} \varepsilon_{r}}
$$

Therefore, equation (S7) transforms to:

$$
\begin{aligned}
& \psi(r)=\psi^{w}+\frac{1}{\varepsilon_{0} \varepsilon_{r}} \int_{r}^{R} d r^{\prime} \frac{1}{r^{\prime}} \int_{0}^{r^{\prime}} d r^{\prime \prime} r^{\prime \prime} q\left(r^{\prime \prime}\right) \\
& =\psi^{w}+\frac{1}{\varepsilon_{0} \varepsilon_{r}}\left[\int_{0}^{r} d r^{\prime} q\left(r^{\prime}\right) r^{\prime} \int_{r}^{R} d r^{\prime \prime} \frac{1}{r^{\prime \prime}}+\int_{r}^{R} d r^{\prime} q\left(r^{\prime}\right) \int_{r^{\prime}}^{R} d r^{\prime \prime} \frac{1}{r^{\prime \prime}}\right. \\
& =\psi^{w}+\frac{1}{\varepsilon_{0} \varepsilon_{r}}\left[\ln \left(\frac{R}{r}\right) \int_{0}^{r} d r^{\prime} q\left(r^{\prime}\right) r^{\prime}+\int_{r}^{R} d r^{\prime} \ln \left(\frac{R}{r^{\prime}}\right) q\left(r^{\prime}\right) r^{\prime}\right]
\end{aligned}
$$

When $r$ approaches zero, by using L'Hospital's rule, we get:

$$
\begin{aligned}
& \psi(0)=\lim _{r \rightarrow 0} \psi(r) \\
& \left.=\psi^{w}+\frac{1}{\varepsilon_{0} \varepsilon_{r}}\left[-\lim _{r \rightarrow 0} \frac{\int_{0}^{r} d r^{\prime} q\left(r^{\prime}\right) r^{\prime}}{\frac{1}{\ln r}}+\lim _{r \rightarrow 0} \int_{r}^{R} d r^{\prime} \ln \left(\frac{R}{r^{\prime}}\right) q\left(r^{\prime}\right) r^{\prime}\right)\right] \\
& \left.=\psi^{w}+\frac{1}{\varepsilon_{0} \varepsilon_{r}}\left[-\lim _{r \rightarrow 0} q\left(r^{\prime}\right)(\ln r \cdot r)^{2}+\int_{0}^{R} d r^{\prime} \ln \left(\frac{R}{r^{\prime}}\right) q\left(r^{\prime}\right) r^{\prime}\right)\right]
\end{aligned}
$$


$=\psi^{w}+\frac{1}{\varepsilon_{0} \varepsilon_{r}} \int_{0}^{R} d r^{\prime} \ln \left(\frac{R}{r^{\prime}}\right) q\left(r^{\prime}\right) r^{\prime}$

\section{References}

1. Pinilla, C.; Del Popolo, M. G.; Lynden-Bell, R. M.; Kohanoff, J. Structure and dynamics of a confined ionic liquid. Topics of relevance to dye-sensitized solar cells. J. Phys. Chem. B 2005, 109(38), 17922-17927.

2. Fitzgerald, J. E.; Sudibandriyo, M.; Pan, Z.; Robinson Jr, R. L.; Gasem, K. A. M. Modeling the adsorption of pure gases on coals with the SLD model. Carbon, 2003, 41(12), 2203-2216.

3. Molecular thermodynamics of nonideal fluids: Lee, L. L. Butterworth-Heinemann, 2016

4. Siderius, D. W.; Gelb, L. D. Extension of the Steele 10-4-3 Potential for Adsorption Calculations in Cylindrical, Spherical, and Other Pore Geometries. J. Chem. Phys. 2011, 135, 084703.

5. Figueroa-Gerstenmaier, S.; Blas, F. J.; Avalos, J. B.; Vega, L. F. Application of the fundamental measure density functional theory to the adsorption in cylindrical pores. $J$. Chem. Phys. 2003, 118(2), 830-842. 